Johan Gobom*, Lucilla Parnetti, Pedro Rosa-Neto, Martin Vyhnalek, Serge Gauthier, Samuela Cataldi, Ondrej Lerch, Jan Laczo, Katerina Cechova, Marcus Clarin, Andrea L. Benet, Tharick A. Pascoal, Neserine Rahmouni, Manu Vandijck, Else Huyck, Nathalie Le Bastard, Jenna Stevenson, Mira Chamoun, Daniel Alcolea, Alberto Lleó, Ulf Andreasson, Marcel M. Verbeek, Giovanni Bellomo, Roberta Rinaldi, Nicholas J. Ashton, Henrik Zetterberg, Katerina Sheardova, Jakub Hort and Kaj Blennow

\title{
Validation of the LUMIPULSE automated immunoassay for the measurement of core AD biomarkers in cerebrospinal fluid
}

https://doi.org/10.1515/cclm-2021-0651

Received January 1, 2021; accepted November 2, 2021; published online November 15, 2021
Abstract

Objectives: The core cerebrospinal fluid (CSF) biomarkers; total tau (tTau), phospho-tau (pTau), amyloid

\begin{abstract}
*Corresponding author: Johan Gobom, Department of Psychiatry and Neurochemistry, Institute of Neuroscience and Physiology, The Sahlgrenska Academy, University of Gothenburg, Gothenburg, Sweden; and Clinical Neurochemistry Laboratory, Sahlgrenska University Hospital, Mölndal, Sweden, E-mail: johan.gobom@neuro.gu.se. https://orcid.org/0000-0001-6193-6193

Lucilla Parnetti, Samuela Cataldi, Giovanni Bellomo and Roberta Rinaldi, Laboratory of Clinical Neurochemistry, Section of Neurology, University of Perugia, Perugia, Italy

Pedro Rosa-Neto and Serge Gauthier, Department of Neurology and Neurosurgery, McGill University Research Centre for Studies in Aging, Douglas Research Institute, Le Centre intégré universitaire de santé et de services sociaux (CIUSSS) de l'Ouest-de-l'île-de-Montréal, Psychiatry and Pharmacology and Therapeutics, McGill University, Montreal, QC, Canada; and Montreal Neurological Institute, Montreal, QC, Canada
\end{abstract}

Martin Vyhnalek, Ondrej Lerch, Jan Laczo and Katerina Cechova, Department of Neurology, Second Medical Faculty, Charles University, Prague, Czech Republic; Motol University Hospital, Prague, Czech Republic; and International Clinical Research Center, St. Anne's University Hospital, Brno, Czech Republic.

https://orcid.org/0000-0002-5845-1854 (K. Cechova)

Marcus Clarin, Ulf Andreasson and Kaj Blennow, Department of Psychiatry and Neurochemistry, Institute of Neuroscience and Physiology, The Sahlgrenska Academy, University of Gothenburg, Gothenburg, Sweden; and Clinical Neurochemistry Laboratory, Sahlgrenska University Hospital, Mölndal, Sweden

Andrea L. Benet, Department of Psychiatry and Neurochemistry, Institute of Neuroscience and Physiology, The Sahlgrenska Academy, University of Gothenburg, Gothenburg, Sweden; and Translational Neuroimaging Laboratory, McGill Centre for Studies in Aging, McGill University, Montreal, QC, Canada

Tharick A. Pascoal, Neserine Rahmouni, Jenna Stevenson and Mira Chamoun, Translational Neuroimaging Laboratory, McGill Centre for Studies in Aging, McGill University, Montreal,

QC, Canada
Manu Vandijck, Else Huyck and Nathalie Le Bastard, Fujirebio Europe N.V., Ghent, Belgium

Daniel Alcolea and Alberto Lleó, Department of Neurology, Memory Unit, Hospital de la Santa Creu i Sant Pau- Biomedical Research Institute Sant Pau-Universitat Autònoma de Barcelona, Barcelona, Spain; and Centro de Investigación Biomédica en Red Enfermedades Neurodegenerativas (CIBERNED), Madrid, Spain

Marcel M. Verbeek, Department of Laboratory Medicine, Radboud University Medical Center, Nijmegen, The Netherlands; and Department of Neurology, Radboud Alzheimer Centre, Radboud University Medical Center, Donders Institute for Brain, Cognition and Behaviour, Nijmegen, The Netherlands

Nicholas J. Ashton, Department of Psychiatry and Neurochemistry, Institute of Neuroscience and Physiology, The Sahlgrenska Academy, University of Gothenburg, Gothenburg, Sweden; Wallenberg Centre for Molecular and Translational Medicine, University of Gothenburg, Gothenburg, Sweden; King's College London, Institute of Psychiatry, Psychology \& Neuroscience, Maurice Wohl Clinical Neuroscience Institute, London, UK; and NIHR Biomedical Research Centre for Mental Health \& Biomedical Research Unit for Dementia at South London \& Maudsley NHS Foundation, London, UK Henrik Zetterberg, Department of Psychiatry and Neurochemistry, Institute of Neuroscience and Physiology, The Sahlgrenska Academy, University of Gothenburg, Gothenburg, Sweden; Clinical Neurochemistry Laboratory, Sahlgrenska University Hospital, Mölndal, Sweden; Department of Neurodegenerative Disease, UCL Institute of Neurology, London, UK; and UK Dementia Research Institute at UCL, London, UK

Katerina Sheardova, Department of Neurology, Second Medical Faculty, Charles University, Prague, Czech Republic; and First Department of Neurology, Faculty of Medicine, Masaryk University and St. Anne's University Hospital, Brno, Czech Republic Jakub Hort, Department of Neurology, Second Medical Faculty, Charles University, Prague, Czech Republic; Motol University Hospital, Prague, Czech Republic; and First Department of Neurology, Faculty of Medicine, Masaryk University and St. Anne's University Hospital, Brno, Czech Republic 
$\beta$ 1-42 (A $\beta$ 1-42), and the $A \beta$ 1-42/A $\beta$ 1-40 ratio have transformed Alzheimer's disease (AD) research and are today increasingly used in clinical routine laboratories as diagnostic tools. Fully automated immunoassay instruments with ready-to-use assay kits and calibrators has simplified their analysis and improved reproducibility of measurements. We evaluated the analytical performance of the fully automated immunoassay instrument LUMIPULSE G (Fujirebio) for measurement of the four core AD CSF biomarkers and determined cutpoints for AD diagnosis.

Methods: Comparison of the LUMIPULSE G assays was performed with the established INNOTEST ELISAs (Fujirebio) for hTau Ag, pTau 181, $\beta$-amyloid 1-42, and with V-PLEX Plus A $\beta$ Peptide Panel 1 (6E10) (Meso Scale Discovery) for $A \beta 1-42 / A \beta 1-40$, as well as with a LC-MS reference method for $A \beta$ 1-42. Intra- and inter-laboratory reproducibility was evaluated for all assays. Clinical cutpoints for $A \beta$ 1-42, tTau, and pTau was determined by analysis of three cohorts of clinically diagnosed patients, comprising $651 \mathrm{CSF}$ samples. For the $\mathrm{A} \beta$ 1-42/A $\beta$ 1-40 ratio, the cutpoint was determined by mixture model analysis of 2,782 CSF samples.

Results: The LUMIPULSE G assays showed strong correlation to all other immunoassays ( $>0.93$ for all assays). The repeatability (intra-laboratory) CVs ranged between 2.0 and $5.6 \%$, with the highest variation observed for $\beta$-amyloid 1-40. The reproducibility (inter-laboratory) CVs ranged between 2.1 and $6.5 \%$, with the highest variation observed for $\beta$-amyloid 1-42. The clinical cutpoints for AD were determined to be $409 \mathrm{ng} / \mathrm{L}$ for total tau, $50.2 \mathrm{ng} / \mathrm{L}$ for pTau $181,526 \mathrm{ng} / \mathrm{L}$ for $\beta$-amyloid 1-42, and 0.072 for the $A \beta 1-42 / A \beta$ 1-40 ratio.

Conclusions: Our results suggest that the LUMIPULSE G assays for the CSF AD biomarkers are fit for purpose in clinical laboratory practice. Further, they corroborate earlier presented reference limits for the biomarkers.

Keywords: Alzheimer's disease; biomarkers; immunoassay; LUMIPULSE; validation.

\section{Introduction}

Alzheimer's disease (AD) is the most common cause of dementia [1]. The neuropathological hallmarks of the disease are amyloid plaques, composed of amyloid beta $(A \beta)$ peptides [2], and intraneuronal neurofibrillary tangles, consisting of hyperphosphorylated tau protein (pTau) [3]. The most abundant form of $A \beta$ in the extracellular plaques is a 42-amino acid peptide denoted $A \beta 1$ 1-42 [4]. $A \beta$ peptides are formed through the cleavage of the transmembrane amyloid precursor protein [5]. Tau in its native form, i.e. without abnormal phosphorylation, is a protein found intracellularly and is involved in the stabilization of the microtubules [6].

In $\mathrm{AD}$, the typical biomarker pattern in cerebrospinal fluid (CSF) is a combination of decreased levels of $A \beta 1-42$ and increased levels of pTau and total tau (tTau) [4]. CSF tTau is measured using assays based on antibodies that detect all tau isoforms, independently of phoysphorylation status [4]. These biomarkers are used extensively in research but have not yet become included in the clinical diagnostic criteria for $\mathrm{AD}$, although clinicians use them already now as support for the diagnosis [7]. The A $\beta$ 1-42 concentration may be normalized to the concentration of the 40-amino acid-long form of beta-amyloid (A $\beta$ 1-40) to obtain the $A \beta 1-42 / A \beta 1-40$ ratio, which has proved to yield improved diagnostic performance compared to $A \beta$ 1-42 alone [8]. While the ratio decreases the impact of preanalytical sources of errors, e.g., adsorption of peptides to CSF collection tubes and pipetting inaccuracy, it has also been suggested that it may serve to compensate for physiological variation in the expression and processing of the amyloid precursor protein $[8,9]$.

Enzyme-linked immunosorbent assays (ELISA), such as the INNOTEST $\beta$-amyloid 1-42, hTau Ag, and PHOSPHOTAU(181P) assays, have for a long time been used to measure the CSF biomarkers. A drawback of manually performed ELISA assays is that they are prone to variability; minor variations in the quality of the reagents, laboratory environment, and execution of the assay protocol may significantly affect the result, thereby making it difficult to obtain consistent results over time and between labs [10]. Such variation has been greatly decreased by the implementation of automated immunoassay platforms with pre-supplied ready-to-use reagents that have become available in recent years from several assay manufacturers.

The LUMIPULSE G (Fujirebio) is an automated system for bead-based immunoassays, which is able to measure all four AD biomarkers; $A \beta$ 1-42, tTau, pTau, and the $A \beta$ 1-42/A $1-40$ ratio.

Clinical validation studies of the LUMIPULSE G assays have shown good correlations in comparisons with other immunoassays, low intra- and inter-assay varitation, and good concordance with clinical diagnosis [11-16], and recent studies reported good concordance between LUMIPULSE G amyloid $\beta$ (1-42) or amyloid $\beta$ (1-42)/total tau ratio, and amyloid PET status [17-20].

In this study, we evaluated the performance of LUMIPULSE $G$ in relation to established immunoassays for measurement of the core AD biomarkers, and for LUMIPULSE G amyloid $\beta$ 1-42, performed a comparison with an 
LC-MS based certified reference method [21]. We also evaluated intra- and inter-laboratory and longitudinal variability, and by analysis of three cohorts of clinically diagnosed patients, determined clinical cutpoints for $A \beta 42$, A $342 / \mathrm{A} 340$, pTau and tTau.

\section{Materials and methods}

\section{LUMIPULSE}

For measurement of amyloid $\beta$ 1-40, amyloid $\beta$ 1-42, total tau and pTau 181, a LUMIPULSE ${ }^{\circledR}$ G600II instrument was used (Fujirebio, Ghent, Belgium). It is a cartridge-based system that uses monoclonal antibody-coated beads for capture and monoclonal antibodies for detection. For amyloid $\beta$ 1-42 and total tau, streptavidin-conjugated alkaline phosphatase (AP) is added after a washing step, which binds to the biotinylated monoclonal antibody. Amyloid $\beta$ 1-40 and pTau 181 , the antibodies are directly conjugated to AP instead, and 3-(2'-spiroadamantane)-4-methoxy-4-(3"-phosphoryloxy) phenyl-1, 2-dioxetane disodium salt (AMPPD) is used as substrate and the resulting luminescence at $477 \mathrm{~nm}$ is measured.

\section{Method comparisons}

Method comparisons were performed at the neurochemistry laboratory, Sahlgrenska University Hospital/Mölndal, using de-identified CSF-samples from the hosptal routine analysis. Samples were selected based on initial INNOTEST results and re-analyzed on the same occasion by LUMIPULSE $G$ and the respective assay. The use of surplus CSF from the routine analysis was approved by the Ethics Committee at the University of Gothenburg (EPN - Gothenburg, Aug 11, 2014).

INNOTEST $\beta$-amyloid (1-42), hTau Ag, and pTau 181 (Fujirebio) assays were performed according to the manufacturer's kit insert instructions as described previously [22]. Samples with biomarker concentrations below the lower limit of quantification (LLoQ) as provided by the manufacturer were excluded ( $\beta$-amyloid (1-42)<225 ng/L, hTau $\mathrm{Ag}<57 \mathrm{ng} / \mathrm{L}$ and pTau $181<20 \mathrm{ng} / \mathrm{L}$ ). MSD electrochemiluminescence assay (MSD) was used according to the manufacturer's instructions for measurement of $A \beta 1-42 / A \beta 1-40$.

LC-MS analysis of A $\beta$ 1-42 was performed using a certified reference method, as previously described [21, 23].

\section{Reproducibility}

CSF samples having low, medium, and high concentrations of the four analytes were analyzed by LUMIPULSE G in three laboratories. The experiments were performed in two separate rounds: in the first round, Total Tau was measured at the University of Gothenburg, Sweden (Lab "a" in Figure 2), at Radboud University, Netherlands (Lab "b"), and at FujiRebio, Belgium (Lab “c”). In the second round, pTau 181, $\beta$-amyloid 1-42, and $\beta$-amyloid 1-40 were measured at Radboud University, FujiRebio, and Sant Pau- Biomedical Research Institute, Spain (Lab "d"). The homogeneity of the sample aliquots was verified by repeated measurments by at the FujiRebio laboratory, Belgium. In each laboratory, the samples were analyzed in triplicate, twice per day over five days. For some measurements, there was insufficient sample volume avaliable; these measurements were then excluded from the comparison. The standard deviations and coefficients of variation (CV) for intra-laboratory repeatability and inter-laboratory reproducibility were calculated according to ISO 5725-2.

Inter- and intra-lab variation were also assessed by analyzing samples within the Alzheimer's Association Quality Control (AA-QC) program, administered at the Clinical Neurochemistry Laboratory at the University of Gothenburg, Sweden [10]. In the AA-QC program, CSF samples with known concentrations of the $\mathrm{AD}$ biomarkers are periodically dispatched to laboratories around the world for method validation to monitor inter-lab variability of $\mathrm{AD}$ biomarkers. For LUMIPULSE G, only results of $\beta$-amyloid 1-42 and total tau were available. Individual CSF samples were analyzed on seven different occasion. For $\beta$-amyloid 1-42 a longitudinal sample consisting of pooled CSF was analysed at seven rounds with 4-19 participating laboratories. For tTau we used data from a pooled CSF sample analysed at four time points with 4-19 participating laboratories in each round.

Long-term consistency of measurments for all four assays, performed on a single LUMIPULSE G instrument was evaluated by analyzing aliquots of two CSF pools; one composed of patient samples with $\mathrm{AD}$-like core biomarker profile, and one with normal biomarker levels. Samples were analyzed 71 times approximately once per week during 18 months in Gothenburg.

\section{Clinical cutpoint determination}

For determination of clinical cutpoints for tTau, pTau, and A 342 , CSF samples from three cohorts were used; from McGill University (Translational Biomarkers for Aging and dementia (TRIAD), Canada), University of Perugia (Italy), and Brno/Praha (Czech Republic). Demographics and biomarker data of the samples are listed in Table 5.

For the TRIAD cohort ( $\mathrm{n}=101)$, lumbar puncture (LP) was performed under local anesthesia, using an 18 ga. "introducer" to penetrate the interspinous ligaments, followed by dural puncture using the 24 ga. Sprotte atraumatic needle. Twenty nine milli-liter of fluid was collected with polypropylene syringes into $10 \mathrm{~mL}$ polypropylene tubes (Sarstedt, part no. 62.9924.294). The first four ml was sent to the clinical laboratory for determination of albumin, total protein, glucose and cells. The remaining $25 \mathrm{~mL}$ was transferred to polypropylene tubes and centrifuged at $20^{\circ} \mathrm{C}$ for $10 \mathrm{~min}$ at $2,200 \times \mathrm{g}$. The CSF was then rapidly frozen for permanent storage at $-80^{\circ} \mathrm{C}$ until analysed on the LUMIPULSE instrument.

The Perugia cohort was sampled at the Center of Memory Disturbances of the University of Perugia. CSF samples were obtained from subjects that were consecutively recruited between January 2012 and June 2016 and followed up for at least two years. The cohort consisted of 58 patients with probable AD diagnosed according to the NIA-AA criteria [24], regardless of CSF biomarker profile, and 37 nondemented controls. All patients underwent a baseline clinical examination by experienced neurologists, detailed neuropsychological assessment including Mini-Mental State Examination (MMSE), blood chemistry, MRI and lumbar puncture (LP). Neurological controls included cognitively normal subjects, with other neurological diseases such as headache, epilepsy and polyneuropathies, who showed no evidence of progression to dementia after at least two years of follow-up. Patients with subjective memory complaints were not included in the control group. CSF samples were collected via LP from 

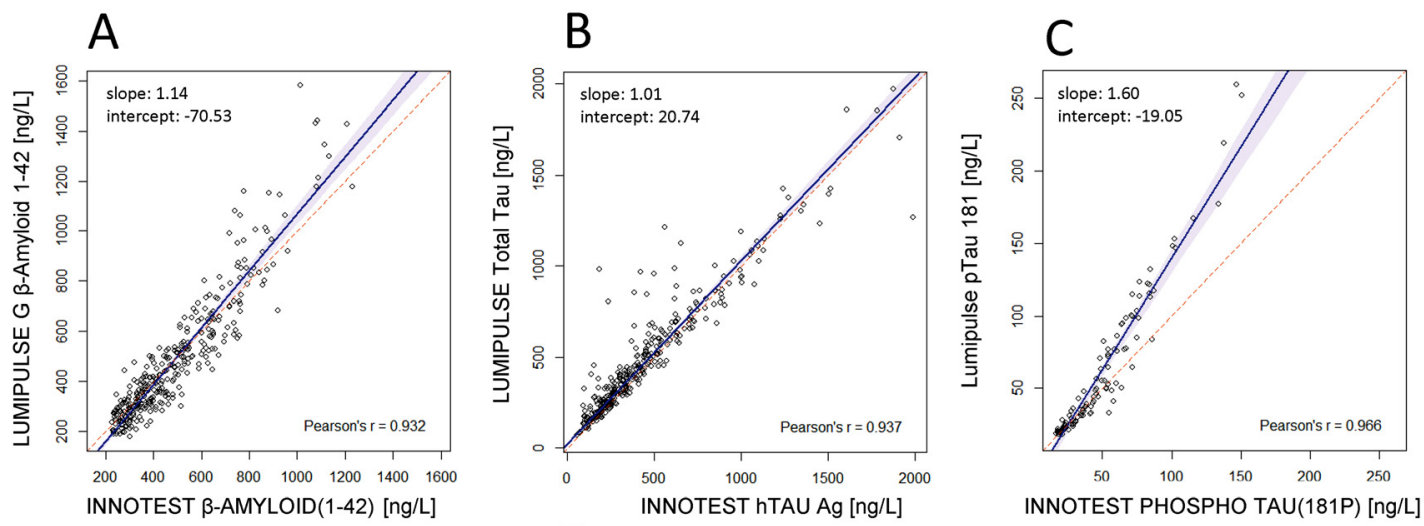

D
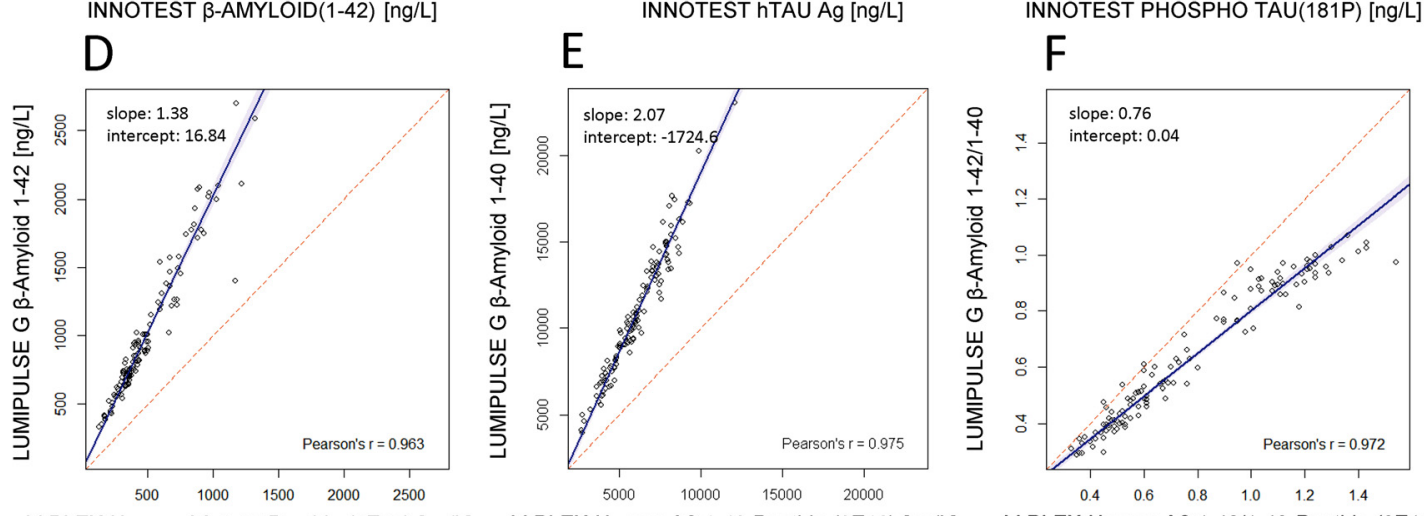

V-PLEX Human A $1-42$ Peptide (6E10) [ng/L]

V-PLEX Human Aß 1-40 Peptide (6E10) [ng/L]

V-PLEX Human Aß 1-42/1-40 Peptide (6E10)

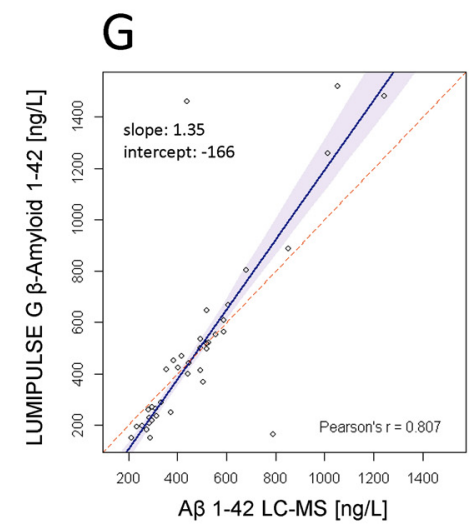

Figure 1: Regression analysis (Passing-Bablok) comparing LUMIPULSE G and: INNOTEST (A) $\beta$-AMYLOID (1-42), (B) hTAU Ag and (C) PHOSPHO TAU(181P); MSD (D) $\beta$-amyloid 1-42, (E) $\beta$-amyloid 1-40, and (F) $10 \times \beta$-amyloid 1-42/1-40; LC-MS (G) $\beta$-amyloid 1-42.

The regression line is indicated in solid blue, and the identity line in dashed orange. The shaded blue area indicates a $95 \%$ confidence interval.

Table 1: Method comparisons.

\begin{tabular}{|c|c|c|c|c|c|}
\hline Biomarker & Reference system & $\mathbf{n}$ & Slope & Intercept & $r$ \\
\hline$\beta$-AMYLOID (1-42) & INNOTEST & 334 & $1.14(1.08,1.19)$ & $-70.53(-92.10,-50.30)$ & 0.932 \\
\hline hTAU Ag & INNOTEST & 354 & $1.01(0.98,1.04)$ & $20.74(12.9,32.0)$ & 0.937 \\
\hline PHOSPHO-TAU (181P) & INNOTEST & 100 & $1.60(1.49,1.70)$ & $-19.05(-24.34,-14.32)$ & 0.966 \\
\hline$A \beta 1-40$ & MSD & 117 & $2.07(2.00,2.18)$ & $-1,724.61(-2,349,-1,256)$ & 0.975 \\
\hline$A \beta 1-42$ & MSD & 117 & $1.38(1.31,1.46)$ & $16.84(-7.22,39.46)$ & 0.978 \\
\hline $10 \times A \beta 1-42 / A \beta 1-40$ & MSD & 117 & $0.76(0.73,0.79)$ & $0.04(0.01,0.06)$ & 0.972 \\
\hline$A \beta 1-42$ & LC-MS & 40 & $1.35(1.24,1.58)$ & $-166(-240.0,-122.2)$ & 0.807 \\
\hline
\end{tabular}

Slope and Intercept denote Passing-Bablok regression parameters for comparisons between LUMIPULSE G and the respective reference system. $\mathrm{R}$, Pearson's regression coefficient. 
A
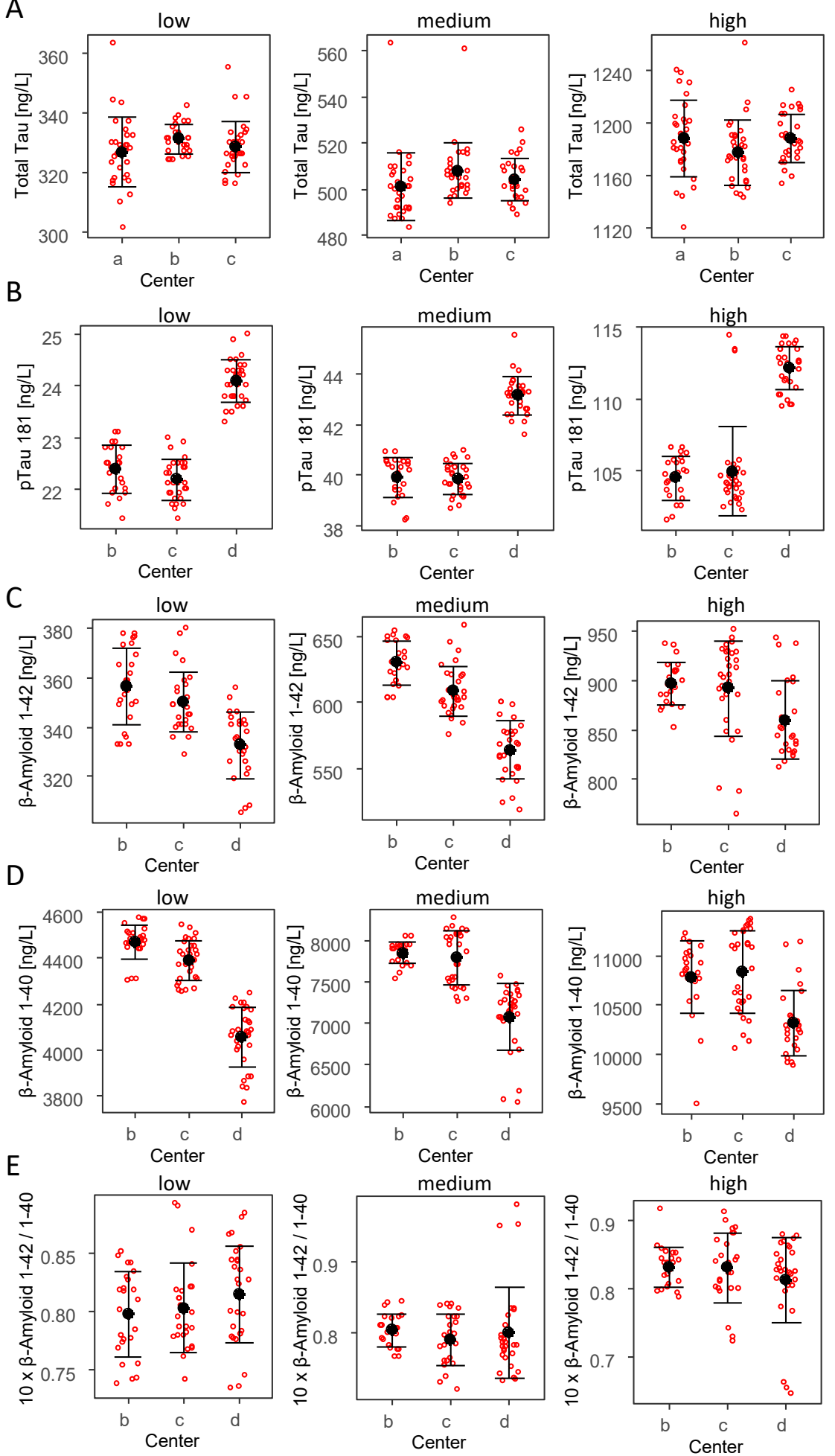

Figure 2: Inter- and intra-lab variation of LUMIPULSE G (A) total tau, (B) pTau 181, (C) $\beta$-amyloid 1-42, (D) $\beta$-amyloid 1-40, and (E) $\beta$-amyloid 1-42/1-40, measured at three (out of four) different laboratories designated a-d.

The black circle indicates value of the mean and the bars represent \pm 1 standard deviation.

8:00 to 10:00 a.m. after overnight fasting, following a standardized procedure [12] and according to international guidelines [25, 26]. CSF $(10-12 \mathrm{~mL})$ was taken from the L3-L4 or L4-L5 interspace, immediately collected in sterile polypropylene tubes (Sarstedt cat. nr. 62.610.201), and gently mixed to avoid possible gradient effects. The samples were centrifuged at $2000 \times \mathrm{g}$ for $10 \mathrm{~min}$ at room temperature, aliquoted $(0.5 \mathrm{~mL})$ in polypropylene tubes (Sarstedt cat. nr. 72.730.007) and stored at $-80^{\circ} \mathrm{C}$ pending analysis.

The Czech cohort was collected within the Czech Brain Aging Study (CBAS) [27]. CBAS is a prospective longitudinal memory clinic- 
Table 2: Inter- and intra-laboratory variation.

\begin{tabular}{|c|c|c|c|c|c|c|c|}
\hline & \multirow[t]{2}{*}{ Analyte concentration } & \multirow[t]{2}{*}{$\mathbf{n}$} & \multirow[t]{2}{*}{ Mean } & \multicolumn{2}{|c|}{$\begin{array}{c}\text { Repeatability } \\
\text { (intra-laboratory) }\end{array}$} & \multicolumn{2}{|c|}{$\begin{array}{l}\text { Reproducibility } \\
\text { (inter-laboratory) }\end{array}$} \\
\hline & & & & SD & CV, \% & SD & $\mathrm{CV}, \%$ \\
\hline \multirow[t]{3}{*}{ Total tau, ng/L } & Low & 88 & 328.7 & 8.95 & 2.72 & 9.06 & 2.76 \\
\hline & Medium & 90 & 504.3 & 12.13 & 2.41 & 12.44 & 2.47 \\
\hline & High & 90 & $1,184.7$ & 24.24 & 2.05 & 24.56 & 2.07 \\
\hline \multirow[t]{3}{*}{ pTau 181, ng/L } & Low & 84 & 22.92 & 0.42 & 1.84 & 1.15 & 5.02 \\
\hline & Medium & 84 & 41.04 & 0.71 & 1.74 & 2.04 & 4.98 \\
\hline & High & 84 & 107.4 & 2.22 & 2.06 & 4.87 & 4.54 \\
\hline \multirow[t]{3}{*}{ Amyloid $\beta$ 1-42, ng/L } & Low & 78 & 346.3 & 13.65 & 3.94 & 18.16 & 5.24 \\
\hline & Medium & 80 & 598.4 & 19.63 & 3.28 & 38.66 & 6.46 \\
\hline & High & 76 & 883.3 & 39.26 & 4.44 & 43.17 & 4.89 \\
\hline \multirow[t]{3}{*}{ Amyloid $\beta 1-40, \mathrm{ng} / \mathrm{L}$} & Low & 84 & $4,293.4$ & 101.6 & 2.37 & 243.3 & 5.67 \\
\hline & Medium & 84 & $7,788.7$ & 4.13 & 5.59 & $7,844.8$ & 1.65 \\
\hline & High & 81 & $10,647.2$ & 378.3 & 3.55 & 469.8 & 4.41 \\
\hline \multirow[t]{3}{*}{$10 \times$ amyloid $\beta$ 1-42/1-40 } & Low & 77 & 0.806 & 0.039 & 4.79 & 0.039 & 4.82 \\
\hline & Medium & 76 & 0.797 & 0.046 & 5.794 & 0.046 & 5.794 \\
\hline & High & 77 & 0.824 & 0.051 & 6.157 & 0.051 & 6.170 \\
\hline
\end{tabular}

Analytical variation is expressed as standard deviation (SD) and coeficcient of variation (CV) of intra-laboratory repeatability and interlaboratory variation, respectively.

A

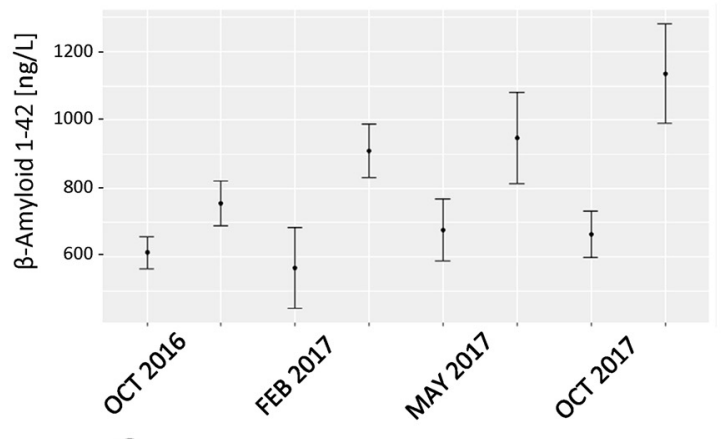

C

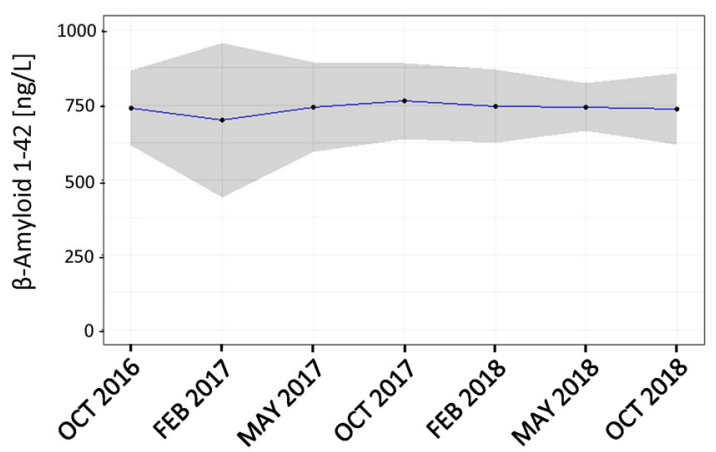

B

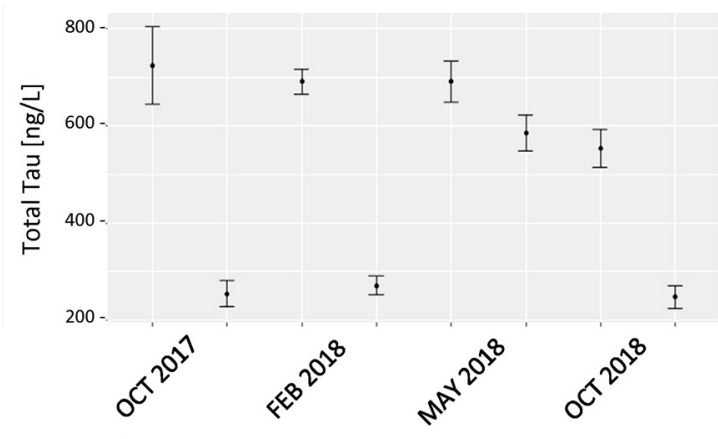

D

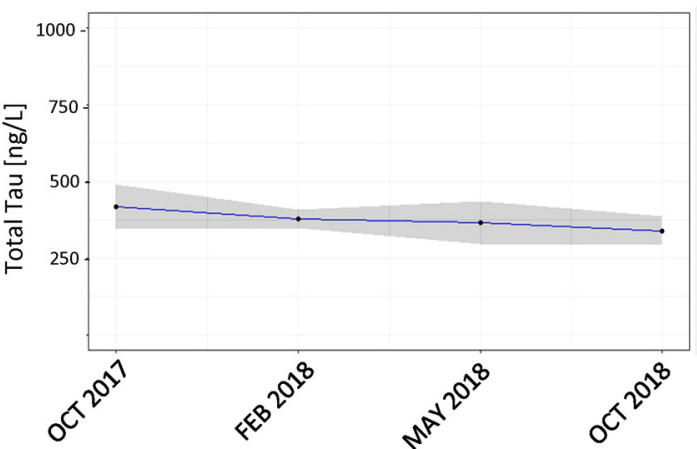

Figure 3: Inter-laboratory variation in the AA-QC program.

(A and B) Variation of (A) $\beta$-amyloid 1-42 and (B) total tau for different samples measured in different rounds. The mean for each round is indicated by a circle and the whiskers indicate \pm 1 standard deviation. (C and D) Variation for (C) $\beta$-amyloid 1-42 and (D) total tau for a longitudinal sample analysed in seven and four rounds, respectively. The shaded gray area indicates a $95 \%$ confidence interval. 
based multicentre study recruiting non-demented adults 55+ years of age. Both CBAS centres in Prague and Brno work as a low-threshold facility; hence, the participants are mostly volunteers who enter by self-referral, with memory complaints expressed by themselves or the family or who were referred by general practitioners, local specialists or the Czech Alzheimer Society to one of the memory clinics. All study participants underwent a standard set of procedures, including neurological and comprehensive neuropsychology examinations, as well as laboratory and vital function assessments. Sociodemographic, personal, pharmacological and family history data were collected. Participants and their informants completed multiple questionnaires about cognitive complaints and lifestyle factors. MRI scans of 1.5 or $3 \mathrm{~T}$ were performed every 24 months or earlier when a participant converted to dementia or progressed towards cognitive impairment at an unusual rate. Genotyping was carried out at baseline. In a subset, CSF and/or amyloid PET was performed. The CBAS is complemented by a biological sample bank linked to data from the CBAS and CBAS Plus cohorts. The cerebrospinal fluid (CSF) collection and storage were carried out according to the widely recognised consensus protocol for the standardisation of CSF collection and biobanking. CSF was collected in polypropylene $10 \mathrm{~mL}$ tubes (Gama, part no. 400 942). Eighteen aliquots of $0.2 \mathrm{~mL} \mathrm{CSF}$ were stored in polypropylene tubes for each participant. All samples were stored at $-80^{\circ} \mathrm{C}$.

The Czech and Italian cohorts were analyzed by LUMIPULSE $\mathrm{G}$ at the respective sites where the samples were collected, while the Canadian cohort and the clinical routine samples used to establish clinical cutpoints for $A \beta 1-42 / A \beta 1-40$ were analyzed at the University of Gothenburg in Sweden. Use of the samples for these studies was granted by the respective local ethics boards: the Czech cohort: No. EK-701/16, Date of EC Session: 25.5.2016; the Italian cohort: Prot. N. 1936908/AV del 09/1012008; the Canadian cohort: REB from Douglas Hospital Research Centre - Montreal - Canada, no. IUSMD-16-60.

Determination of clinical cutpoint for $A \beta$ 1-42/A $\beta$ 1-40 was based on mixture model analysis of data from 2,782 de-identified patient samples analyzed at the Neurochemistry Laboratory at Sahlgrenska University Hospital between December 2019 - October 2020.

\section{Statistical analysis}

Statistical data analysis was performed in R (version 3.6.1; http:// www.R-project.org/). Passing-Bablok regression analysis was performed using the R package $m c r$ (https://CRAN.R-project.org/package $=$ mcr). Clinical cutpoints for tTau, pTau, and A $\beta 42$ were determined by ROC curve analysis, using the ROC01 algorithm [28, 29], implemented in the R package "pROC" [30]. For A $\beta 42 / A \beta 40$, the cutpoint was determined by mixture model analysis, using the expectation-maximization (EM) algorithm for mixtures of normal distributions (normalmixEM), implemented in the R package "mixtools" [31].

\section{Results}

\section{Method comparisons}

The LUMIPULSE G assays $\beta$-amyloid 1-42, $\beta$-amyloid 1-40, total tau, and pTau 181 were compared to the corresponding

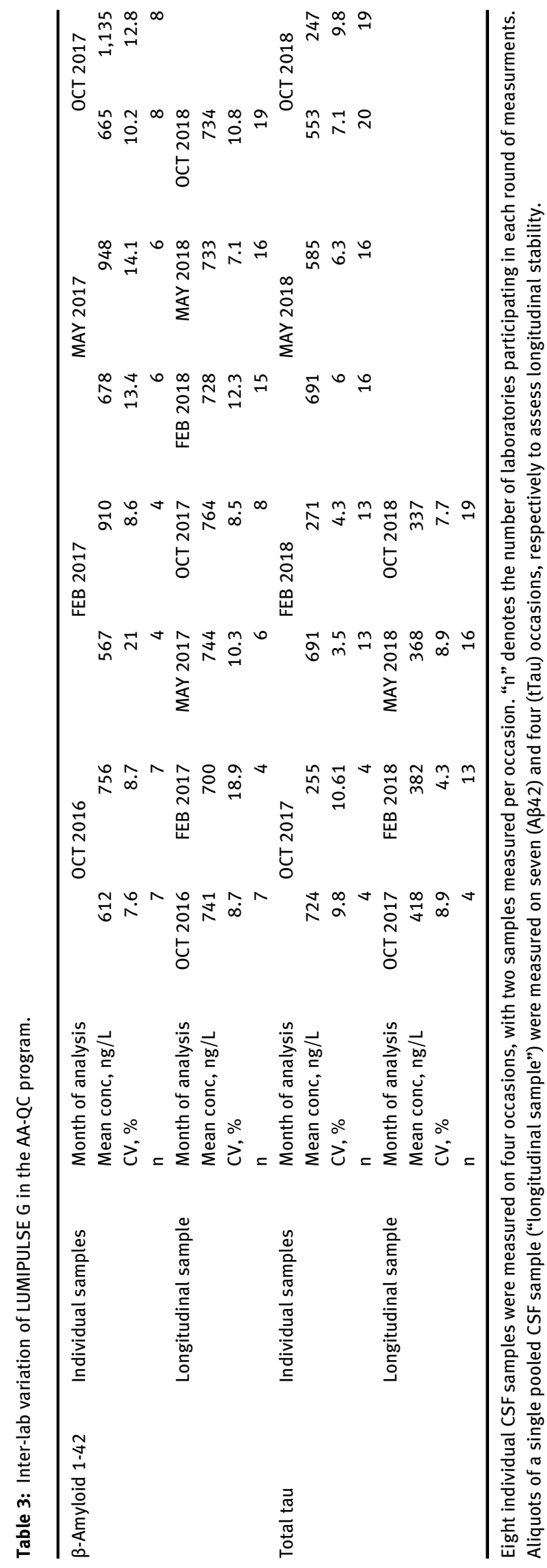


INNOTEST assays, $\beta$-AMYLOID (1-42), $\beta$-AMYLOID (1-40), hTAU Ag, and PHOSPHO TAU (181P) (Figure 1A-C); to MSD (V-PLEX Plus $A \beta$ Peptide Panel 1 (6E10)) for $A \beta 1$ 1-42, $A \beta 40$, and $A \beta$ 1-42/A 340 (Figure 1D-F); an LC-MS reference method for $A \beta$ 1-42 (Figure $1 G$ ), using regression analysis. The results are summarized in Table 1. The LUMIPULSE G assays showed strong correlation to all other immunoassays ( $>0.93$ for all assays). The results for LUMIPULSE $\mathrm{G}$ amyloid 1-42 and total tau were similar to the corresponding INNOTEST ELISA assays, with small intercept and slope close to one in the correlation plots. LUMIPULSE pTau 181 also had a small intercept but a positive bias (slope=1.6) compared to INNOTEST PHOSPHO TAU(181P). Compared to the MSD V-PLEX assays for $A \beta 1-42$ and A $\beta 1-40$, LUMIPULSE $G$ also gave significantly higher values for both $\beta$-amyloid 142 (slope=1.38, intercept=16.8) and $\beta$-amyloid $1-40$ (slope $=2.07$, intercept $=-1,724.6$ ), and approximatley $20 \%$ lower $A \beta 1$ 1-42/A $\beta$ 1-40 ratio. LUMIPULSE G $\beta$-amyloid 1-42, which has been adjusted according to an LC-MS based reference method [21, 23], gave similar values to LC-MS in the range of $400-800 \mathrm{ng} / \mathrm{L}$ but showed a slight negative bias at lower concentrations, and a positive bias at higher concentrations.

\section{Reproducibility}

The intra-laboratory repeatability CVs (Figure 2, Table 2). were below $6 \%$ for all four analytes, with the largest variation for a single analyte was observed for $\beta$-amyloid 1-40 (6.2\%), while the $\beta$-amyloid 1-42/1-40 ratio had a CV of $6.2 \%$. Inter-laboratory reproducibility CVs were similar in magnitude, with the largest CV observed for $\beta$-amyloid 1-42 (6.5\%).

Reproducibility was also studied within the AA-QC program. $\beta$-amyloid 1-42 and total tau were measured in (different) CSF samples, analysed on seven occasions in different laboratories (Figure 3A, B, Table 3). The interlaboratory variation varied significantly between measurement rounds, from $7.6-21 \%$ for $\beta$-amyloid 1-42, and from 3.5-10.6\% for Total Tau. Longitudinal measurement of a single sample indicated no significant drift over time for $\beta$-amyloid 1-42 (Figure 3C, Table 3), but again high CV variation between rounds (7.1-18.9\%). For total tau (Figure 3D, Table 3), there was a slight downward trend over time, with the last measurement $20 \%$ lower than the first, while the CVs were more uniform between the measurement rounds (4.3-8.9\%) compared to $\beta$-amyloid 1-42.

Long-term assay stability was also assessed by measuring aliquots of two CSF sample pools, analyzed on a single LUMIPULSE G instrument in Gothenburg twice per week over an 18-month period (Table 4). For the AD pool (CSF Pool 2), the CV:s for all analytes were below $4 \%$, while for the normal pool (CSF Pool 1) they were slightly higher, but for all analytes within $8 \%$.

\section{Diagnostic performance and cut-off values}

To determine the diagnostic performance of the LUMIPULSE G $\beta$-amyloid 1-42, $\beta$-amyloid 1-42/1-40, total tau, and pTau 181 assays and establish cut-off values for use in a clinical setting, we analyzed CSF from three cohorts of AD patients and non-demented controls (Table 5). Cut-off values were determined by ROC curve analysis of data from all cohorts combined. Histograms depicting the distributions of the biomarker concentrations in the groups and ROC curves are shown in Figure 4, and the established cut-off values, sensitivities and specificities are listed in Table 6, including also values for each cohort separately. The optimal cut-off values were $<409 \mathrm{ng} / \mathrm{L}$ för total tau, $<50.2 \mathrm{ng} / \mathrm{L}$ for pTau 181, and $>526 \mathrm{ng} / \mathrm{L}$ for $\beta$-amyloid 1-42. Because of the high sensitivity and specificity of the $A \beta 42 / A \beta 40$ ratio, this biomarker displays a clear bimodal distribution in patient populations. Therefore, it was possible to use an alternative approach to determining the cut-off value, by using mixture model analysis of data from a large number of patient CSF samples $(n=2,782)$ analyzed in a clinical routine laboratory setting (Figure 5, Table 6), resulting in a cut-off of $>0.72$.

Table 4: Longitudinal stability of the LUMIPULSE G assays on a single instrument.

\begin{tabular}{rlrrrrr}
\hline & & Amyloid $\boldsymbol{\beta}$ 1-40, ng/L & Amyloid $\boldsymbol{\beta}$ 1-42, ng/L & 10 $\times$ amyloid $\boldsymbol{\beta}$ 1-42/1-40 & Tau, ng/L & pTau, ng/L \\
\hline CSF pool 1 & Mean: & $6,669.2$ & 510.2 & 0.76 & 335.3 & 27.0 \\
& CV, \% & 7.4 & 8.0 & 4.36 & 5.2 & 4.2 \\
CSF pool 2 & Mean: & $11,921.5$ & 467.2 & 0.39 & 848.2 & 108.1 \\
& CV, \% & 3.0 & 3.3 & 3.57 & 3.5 \\
\hline
\end{tabular}

Aliquots of two CSF pools; one with normal (Pool 1) and one with AD-type (Pool 2) core biomarker profile, were measured once a week over 18 months $(n=71)$ at the Neurochmeistry Laboratory in Gothenburg. 


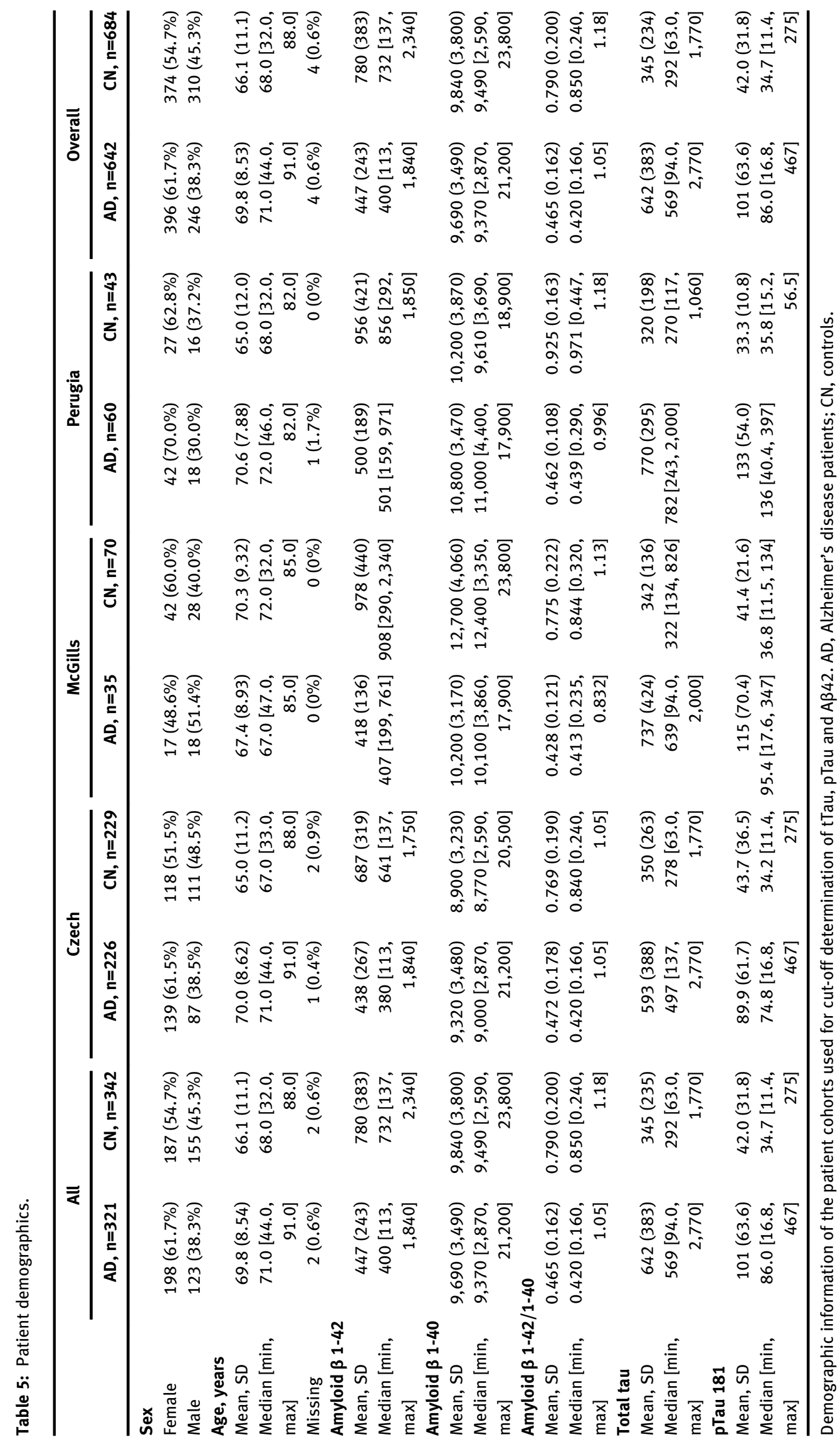



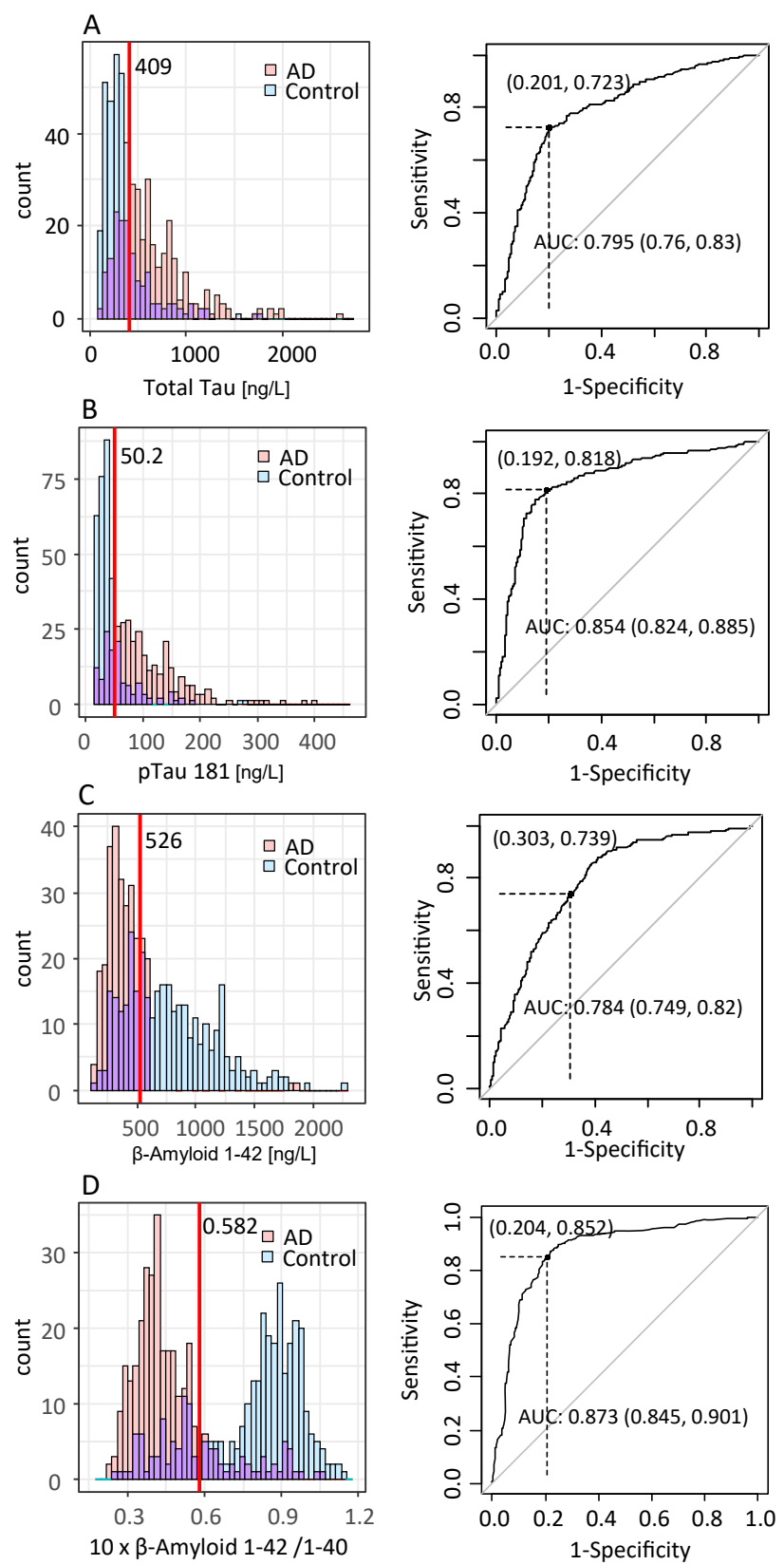

Figure 4: Diagnostic performance of LUMIPULSE and cutpoints. Histograms and ROC curves for (A) tTau, (B) pTau 181, (C) $\beta$-amyloid 1-42, (D) $10 \times \beta$-Amyloid 1-42/1-40 for separating Alzheimer patients and controls, in three combined cohorts.

Total tau and pTau 181 had AUC:s of 80 and $86 \%$, respectively, with sensitivity and specificity of $72 \% / 80 \%$ for total tau of, and $82 \% / 81 \%$ for pTau 181 . $\beta$-amyloid $1-42$ had AUC of $79 \%$ with sensitivity and specificity figures of $85 \% / 84 \%$. For amyloid 1-42/1-40, the sensitivity and specificity, as determined by mixture modeling [31], was $92 \% / 71 \%$.

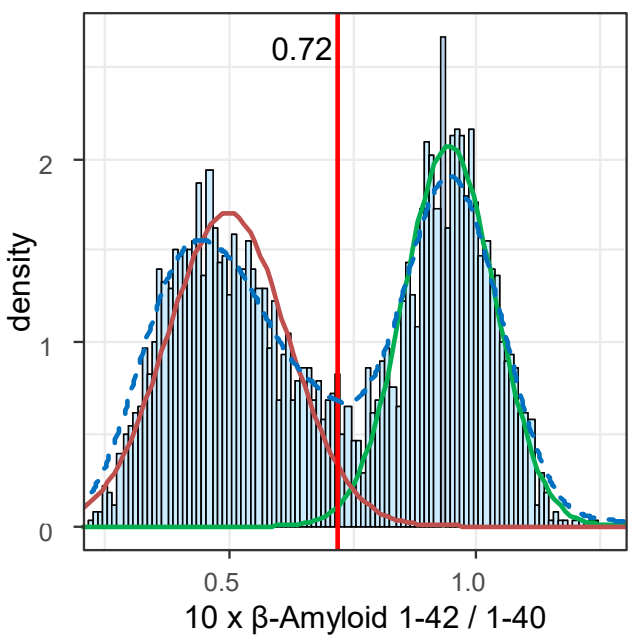

Figure 5: Mixture model analysis of $\beta$-amyloid 1-42/1-40 of data from patient CSF samples $(n=2,782)$ analyzed in a routine laboratory setting.

\section{Discussion}

In the present study, we show good diagnostic performance for LUMIPULSE $G \quad \beta$-amyloid 1-42, $\beta$-amyloid 1-42/40, total tau and pTau 181. All LUMIPULSE G assays show strong linear correlation with INNOTEST amyloid $\beta 1-42$, hTAU Ag and pTau 181, and with MSD for A $\beta 42 / A \beta 40$, as well as good correlation with the LC-MS reference method for $A \beta 1$ 1-42. A slight deviation from linearity can be seen for pTau 181, for which data points in the lower range, below $50 \mathrm{ng} / \mathrm{L}$, appear to follow a regression line with a more shallow slope. Notably, this feature is also visible in the control group data in a recent study by Leitao et al. [14].

Two previous studies have reported large bias between the INNOTEST and LUMIPULSE G $\beta$-amyloid 1-42 assays $[15,16]$ but since December 2018, the LUMIPULSE G assay has been adjusted to harmonize the measurements to the certified reference materials (CRMs) for $A \beta$ 1-42 from the International Federation of Clinical Chemistry and Laboratory Medicine (IFCC). The previous values, as measured before the update, are divided by 1.46 in order to be harmonized with the CRM. This translates to a decrease of about $31.5 \%$, which to a great extent explains the bias observed in previous studies.

The CSF A 1-42/1-40 ratio correlates well between LUMIPULSE G and MSD. However, if the individual concentrations of $A \beta \quad 1-40$ and $A \beta \quad$ 1-42 are compared for correlation, there is an approximate two-fold higher concentration of $A \beta$ 1-40 in LUMIPULSE. This discrepancy highlights the need for a CRM for $\mathrm{A} \beta 40$ to harmonize results between assay platforms. 
Table 6: Diagnostic performance and cut-points of LUMIPULSE G.

\begin{tabular}{|c|c|c|c|c|c|}
\hline Analyte & Cohort & ROC AUC & Sensitivity & Specificity & Cut-off value \\
\hline \multirow[t]{4}{*}{ Total tau } & Czech & 0.76 & $0.71(0.64,0.77)$ & $0.72(0.65,0.77)$ & $<368 \mathrm{ng} / \mathrm{L}$ \\
\hline & Perugia & 0.92 & $0.95(0.86,0.99)$ & $0.91(0.78,0.97)$ & $<431 \mathrm{ng} / \mathrm{L}$ \\
\hline & McGills & 0.87 & $0.86(0.7,0.95)$ & $0.77(0.66,0.86)$ & $<412 \mathrm{ng} / \mathrm{L}$ \\
\hline & Combined & 0.8 & $0.72(0.67,0.77)$ & $0.80(0.75,0.84)$ & $<409 \mathrm{ng} / \mathrm{L}$ \\
\hline \multirow[t]{4}{*}{ pTau 181} & Czech & 0.81 & $0.76(0.7,0.82)$ & $0.80(0.75,0.85)$ & $<50.2 \mathrm{ng} / \mathrm{L}$ \\
\hline & Perugia & 0.99 & $0.97(0.88,1)$ & $1(0.92,-)$ & $<58.3 \mathrm{ng} / \mathrm{L}$ \\
\hline & McGills & 0.92 & $0.86(0.7,0.95)$ & $0.89(0.79,0.95)$ & $<61 \mathrm{ng} / \mathrm{L}$ \\
\hline & Combined & 0.86 & $0.82(0.77,0.86)$ & $0.81(0.77,0.85)$ & $<50.2 \mathrm{ng} / \mathrm{L}$ \\
\hline \multirow[t]{4}{*}{$\beta$-Amyloid 1-42 } & Czech & 0.76 & $0.77(0.71,0.83)$ & $0.67(0.61,0.73)$ & $>512 \mathrm{ng} / \mathrm{L}$ \\
\hline & Perugia & 0.83 & $0.78(0.66,0.88)$ & $0.81(0.67,0.92)$ & $>593 \mathrm{ng} / \mathrm{L}$ \\
\hline & McGills & 0.91 & $0.91(0.77,0.98)$ & $0.76(0.64,0.85)$ & $>573 \mathrm{ng} / \mathrm{L}$ \\
\hline & Combined & 0.79 & $0.74(0.69,0.79)$ & $0.70(0.65,0.75)$ & $>526 \mathrm{ng} / \mathrm{L}$ \\
\hline \multirow[t]{4}{*}{$10 \times \beta$-Amyloid 1-42/40 } & Czech & 0.86 & $0.81(0.75,0.86)$ & $0.79(0.73,0.84)$ & $>0.56$ \\
\hline & Perugia & 0.98 & $0.93(0.84,0.98)$ & $0.95(0.84,0.99)$ & $>0.61$ \\
\hline & McGills & 0.88 & $0.91(0.77,0.98)$ & $0.8(0.69,0.89)$ & $>0.54$ \\
\hline & Combined & 0.58 & $0.85(0.81,0.89)$ & $0.80(0.75,0.84)$ & $>0.58$ \\
\hline $10 \times \beta$-Amyloid 1-42/40 & Gothenburg & Mixture model analysis & 0.92 & 0.71 & $>0.72$ \\
\hline
\end{tabular}

ROC curve analysis and cut-point determination for tTau, $p T a u, A \beta 42$, and $A \beta 42 / A \beta 40$ based on data recorded from three cohorts of $A D$ patients and controls, for each conhort separately as well as combined. For $A \beta 42 / A \beta 40$, an alternative method for cut-point determination was used, based on mixture model analysis of clinical patient data from Gothenburg.

An important aspect of immunoassay platforms for diagnostic use is the ability to generate reproducible results over time and between labs. In the past, the limited ability of many immunoassay methods for the AD biomarkers has made it difficult to compare results reported in different studies, with reproducibility CVs of over 30\% described [32]. Our results show remarkably similar variation for repeatability and reproducibility comparisons, with reproducibility CVs ranging between 5.4 and $6.5 \%$ for A 342 and between 2.1 and $2.8 \%$ for tTau. These results indicate that the use of an automated system with presupplied reagents in a closed cartridge format can greatly improve reproducibility of results between labs.

Data from the AA-QC program show a wide spread of reproducibility $\mathrm{CVs}$ when comparisons were performed, ranging between 7.6 and $21 \%$ for $\mathrm{A} \beta 42$ and $3.5-10.6 \%$ for tTau. A possible reason for this difference is that the reproducibility study was performed under optimal conditions, using the same calibrator and kit lots in all labs and analyzing the samples as part of a study. In the AA-QC program, in contrast, the samples may be measured with different reagent lots and are handled as in clinical routine, where analytical errors are more likely to occur. The longitudinal sample of pooled CSF showed no deviation over time for $\mathrm{A} \beta 42$ or tTau. At this point, longitudinal data is not available for LUMIPULSE G pTau 181 or $\beta$-Amyloid 1-40, as they were only recently included in the program.

Cut-off values were determined in a previous study by Leitao et al. For pTau, the values established in our study are almost identical to those of Leiato et al. (50.2 vs. $50.6 \mathrm{ng} / \mathrm{L}$ ). The cut-points for A $\beta 42$ (526 vs. $543 \mathrm{ng} / \mathrm{L}$ ) and $\mathrm{A} 342 / \mathrm{A} 440$ ( 0.72 vs. 0.68 ) are also similar, whereas that of tTau is $22 \%$ higher ( $409 \mathrm{vs.} 335 \mathrm{ng} / \mathrm{L}$ ).

The histogram in Figure 4D shows that there are several subjects in the control group that have low $\mathrm{A} \beta 42 / \mathrm{A} \beta 40$ ratio. These are possibly individuals with incipient amyloid pathology who do not yet manifest AD symptoms. In the ROC curve analysis, they may lead to underestimation of the optimal cutpoint. For $A \beta 42 / A \beta 40$, the a clear bimodal distribution made it possible to use mixture model analysis to calculate the cutpoint, thus resulting in a slightly higher value compared to that obtained by ROC curve analysis.

In conclusion, the results presented here suggest that the fully automated LUMIPULSE assays for the CSF AD biomarkers are fit for purpose in clinical laboratory practice. Further, they corroborate earlier presented reference limits for the biomarkers.

Research funding: Brno team is supported by the project no. LQ1605 from the National Program of Sustainability II (MEYS CR) and Praha team is supported by Ministry of Health of the Czech Republic, grant no. 19-04-00560. MMV is supported by the BIONIC project (nr. 733050822), which has been made possible by ZonMW (part of the Dutch national 'Deltaplan for Dementia'; zonmw.nl/dementiaresearch), and by grants from the Selfridges Group Foundation, and National Institutes of Health, USA [grant number 5R01NS10414702]. JG is supported by Alzheimerfonden (AF-930934), 
Åhléns-stiftelsen, and Stiftelsen för Gamla tjänarinnor. HZ is a Wallenberg Scholar supported by grants from the Swedish Research Council (\#2018-02532), the European Research Council (\#681712), Swedish State Support for Clinical Research (\#ALFGBG-720931), the Alzheimer Drug Discovery Foundation (ADDF), USA (\#201809-2016862), the European Union's Horizon 2020 research and innovation programme under the Marie Skłodowska-Curie grant agreement No 860197 (MIRIADE), and the UK Dementia Research Institute at UCL. KB is supported by the Swedish Research Council (\#2017-00915), the Alzheimer Drug Discovery Foundation (ADDF), USA (\#RDAPB-2018092016615), the Swedish Alzheimer Foundation (\#AF-742881), Hjärnfonden, Sweden (\#FO2017-0243), the Swedish State Under the Agreement Between the Swedish Government and the County Councils, the ALF-Agreement (\#ALFGBG-715986), and European Union Joint Program for Neurodegenerative Disorders (JPND2019-466-236). PRN is supported by the Canadian Institutes of Health Research (CIHR) [MOP-11-51-31; RFN 152985, 159815, 162303], Canadian Consortium of Neurodegeneration and Aging (CCNA; MOP-11-51-31 -team 1), Weston Brain Institute, the Alzheimer's Association [NIRG-12-92090, NIRP-12-259245], Brain Canada Foundation (CFI Project 34874; 33397), the Fonds de Recherche du Québec - Santé (FRQS; Chercheur Boursier, 2020-VICO279314).

Author contributions: All authors have accepted responsibility for the entire content of this manuscript and approved its submission.

Competing interests: JH has served at scientific advisory board for Alzheon, Agora, Alzheimerchain, Axon, Biogen and has given lectures in symposia sponsored by Schwabe, Lundbeck, Egis, and GE. KS has served at scientific advisory board for Biogen and Alzheimerchain and has given lectures in symposia sponsored by Schwabe and Lundbeck. HZ has served at scientific advisory boards for Denali, Roche Diagnostics, Wave, Samumed, Siemens Healthineers, Pinteon Therapeutics and $\operatorname{CogRx}$, has given lectures in symposia sponsored by Fujirebio, Alzecure and Biogen, and is a co-founder of Brain Biomarker Solutions in Gothenburg AB (BBS), which is a part of the GU Ventures Incubator Program (outside submitted work). KB has served as a consultant, at advisory boards, or at data monitoring committees for Abcam, Axon, Biogen, JOMDD/ Shimadzu. Julius Clinical, Lilly, MagQu, Novartis, Roche Diagnostics, and Siemens Healthineers, and is a co-founder of Brain Biomarker Solutions in Gothenburg AB (BBS), which is a part of the GU Ventures Incubator Program (outside submitted work). PRN is consultant for CERVEAU radiopharmaeuticals. AL has served as a consultant or at advisory boards for Fujirebio-Europe, Roche, Biogen, and Nutricia and has a patent W02019175379 A1 Markers of synaptopathy in neurodegenerative disease issued. DA has served as a consultant or at advisory boards for Krka Farmacéutica S.L., Fujirebio-Europe, Roche Diagnostics, Zambon S.A.U., Esteve Pharmaceuticals S.A. and Nutricia and has a patent WO2019175379 A1 Markers of synaptopathy in neurodegenerative disease issued.

Informed consent: Informed consent was obtained from all individuals included in this study.

Ethical approval: Use of the samples for these studies was granted by the respective local ethics boards: Czech cohort: No. EK-701/16, Date of EC Session: 25.5.2016 Italian cohort : Prot. N. 1936908/AV del 09/1012008.

\section{References}

1. Blennow K, de Leon MJ, Zetterberg H. Alzheimer's disease. Lancet 2006;368:387-403.

2. Masters CL, Simms G, Weinman NA, Multhaup G, McDonald BL, Beyreuther K. Amyloid plaque core protein in Alzheimer disease and down syndrome. Proc Natl Acad Sci USA 1985;82:4245-9.

3. Grundke-Iqbal I, Iqbal K, Tung YC, Quinlan M, Wisniewski HM, Binder LI. Abnormal phosphorylation of the microtubuleassociated protein tau (tau) in Alzheimer cytoskeletal pathology. Proc Natl Acad Sci USA 1986;83:4913-7.

4. Blennow K, Hampel H, Weiner M, Zetterberg H. Cerebrospinal fluid and plasma biomarkers in Alzheimer disease. Nat Rev Neurol 2010;6:131-44.

5. Barthet G, Jorda-Siquier T, Rumi-Masante J, Bernadou F, Muller U, Mulle C. Presenilin-mediated cleavage of APP regulates synaptotagmin-7 and presynaptic plasticity. Nat Commun 2018; 9:4780.

6. Grundke-Iqbal I, Iqbal K, Quinlan M, Tung YC, Zaidi MS, Wisniewski HM. Microtubule-associated protein tau. A component of Alzheimer paired helical filaments. J Biol Chem 1986;261:6084-9.

7. Cognat E, Mouton Liger F, Troussiere AC, Wallon D, Dumurgier J, Magnin E, et al. What is the clinical impact of cerebrospinal fluid biomarkers on final diagnosis and management in patients with mild cognitive impairment in clinical practice? Results from a nation-wide prospective survey in France. BMJ Open 2019;9: e026380.

8. Lewczuk P, Lelental N, Spitzer P, Maler JM, Kornhuber J. Amyloidbeta $42 / 40$ cerebrospinal fluid concentration ratio in the diagnostics of Alzheimer's disease: validation of two novel assays. J Alzheimer's Dis 2015;43:183-91.

9. Janelidze S, Zetterberg H, Mattsson N, Palmqvist S, Vanderstichele $\mathrm{H}$, Lindberg $\mathrm{O}$, et al. CSF Abeta42/Abeta40 and Abeta42/Abeta38 ratios: better diagnostic markers of Alzheimer disease. Ann Clin Transl Neurol 2016;3:154-65.

10. Mattsson N, Andreasson U, Persson S, Arai H, Batish SD, Bernardini S, et al. The Alzheimer's Association external quality control program for cerebrospinal fluid biomarkers. Alzheimer's Dementia 2011;7:386-95.e6. 
11. Bayart JL, Hanseeuw B, Ivanoiu A, van Pesch V. Analytical and clinical performances of the automated Lumipulse cerebrospinal fluid Abeta 42 and T-tau assays for Alzheimer's disease diagnosis. J Neurol 2019;266:2304-11.

12. Chiasserini D, Biscetti L, Farotti L, Eusebi P, Salvadori N, Lisetti V, et al. Performance evaluation of an automated ELISA system for Alzheimer's disease detection in clinical routine. J Alzheimer's Dis 2016;54:55-67.

13. Dakterzada F, Lopez-Ortega R, Arias A, Riba-Llena I, Ruiz-Julian $M$, Huerto $R$, et al. Assessment of the concordance and diagnostic accuracy between elecsys and Lumipulse fully automated platforms and innotest. Front Aging Neurosci 2021; 13:604119.

14. Leitao MJ, Silva-Spinola A, Santana I, Olmedo V, Nadal A, Le Bastard N, et al. Clinical validation of the Lumipulse $\mathrm{G}$ cerebrospinal fluid assays for routine diagnosis of Alzheimer's disease. Alzheimer's Res Ther 2019;11:91.

15. Paciotti S, Sepe FN, Eusebi P, Farotti L, Cataldi S, Gatticchi L, et al. Diagnostic performance of a fully automated chemiluminescent enzyme immunoassay for Alzheimer's disease diagnosis. Clin Chim Acta 2019;494:74-8.

16. Zecca C, Brescia V, Piccininni M, Capozzo R, Barone R, Barulli MR, et al. Comparative evaluation of two immunoassays for cerebrospinal fluid beta-amyloid1-42 measurement. Clin Chim Acta 2019;493:107-11.

17. Alcolea D, Pegueroles J, Munoz L, Camacho V, Lopez-Mora D, Fernandez-Leon A, et al. Agreement of amyloid PET and CSF biomarkers for Alzheimer's disease on Lumipulse. Ann Clin Transl Neurol 2019;6:1815-24.

18. Kaplow J, Vandijck M, Gray J, Kanekiyo M, Huyck E, Traynham CJ, et al. Concordance of Lumipulse cerebrospinal fluid t-tau/ Abeta42 ratio with amyloid PET status. Alzheimer's Dementia 2020;16:144-52.

19. Keshavan A, Wellington H, Chen Z, Khatun A, Chapman M, Hart M, et al. Concordance of CSF measures of Alzheimer's pathology with amyloid PET status in a preclinical cohort: a comparison of Lumipulse and established immunoassays. Alzheimer's Dementia 2020;12:e12097.

20. Moon S, Kim S, Mankhong S, Choi SH, Vandijck M, Kostanjevecki $V$, et al. Alzheimer's cerebrospinal biomarkers from Lumipulse fully automated immunoassay: concordance with amyloid-beta PET and manual immunoassay in Koreans: CSF AD biomarkers measured by Lumipulse in Koreans. Alzheimer's Res Ther 2021; 13:22.
21. Leinenbach A, Pannee J, Dulffer T, Huber A, Bittner T, Andreasson $\mathrm{U}$, et al. Mass spectrometry-based candidate reference measurement procedure for quantification of amyloid-beta in cerebrospinal fluid. Clin Chem 2014;60:987-94.

22. Le Bastard N, Aerts L, Sleegers K, Martin JJ, Van Broeckhoven C, De Deyn PP, et al. Longitudinal stability of cerebrospinal fluid biomarker levels: fulfilled requirement for pharmacodynamic markers in Alzheimer's disease. J Alzheimer's Dis 2013;33:807-22.

23. Blennow K, Mattsson N, Scholl M, Hansson O, Zetterberg H. Amyloid biomarkers in Alzheimer's disease. Trends Pharmacol Sci 2015;36:297-309.

24. McKhann GM, Knopman DS, Chertkow H, Hyman BT, Jack CR Jr., Kawas $\mathrm{CH}$, et al. The diagnosis of dementia due to Alzheimer's disease: recommendations from the National Institute on Aging-Alzheimer's Association Workgroups on diagnostic guidelines for Alzheimer's disease. Alzheimer's Dementia 2011;7: 263-9.

25. del Campo M, Mollenhauer B, Bertolotto A, Engelborghs S, Hampel H, Simonsen AH, et al. Recommendations to standardize preanalytical confounding factors in Alzheimer's and Parkinson's disease cerebrospinal fluid biomarkers: an update. Biomarkers Med 2012;6:419-30.

26. Teunissen CE, Petzold A, Bennett JL, Berven FS, Brundin L, Comabella $\mathrm{M}$, et al. A consensus protocol for the standardization of cerebrospinal fluid collection and biobanking. Neurology 2009;73:1914-22.

27. Sheardova K, Vyhnalek M, Nedelska Z, Laczo J, Andel R, Marciniak $R$, et al. Czech Brain Aging Study (CBAS): prospective multicentre cohort study on risk and protective factors for dementia in the Czech Republic. BMJ Open 2019;9:e030379.

28. Metz CE. Basic principles of ROC analysis. Semin Nucl Med 1978; 8:283-98.

29. Vermont J, Bosson JL, Francois P, Robert C, Rueff A, Demongeot J. Strategies for graphical threshold determination. Comput Methods Progr Biomed 1991;35:141-50.

30. Robin X, Turck N, Hainard A, Tiberti N, Lisacek F, Sanchez JC, et al. pROC: an open-source package for R and S+ to analyze and compare ROC curves. BMC Bioinf 2011;12:77.

31. Benaglia T, Chauveau D, Hunter DR, Young DS. mixtools: an R package for analyzing finite mixture models. J Stat Software 2009;32:1-29.

32. Mattsson N, Zetterberg $\mathrm{H}$, Blennow K. Lessons from multicenter studies on CSF biomarkers for Alzheimer's disease. Int J Alzheimer's Dis 2010;2010:610613. 